ISSN 0103-8478

\title{
Reconhecimento da prole por operárias companheiras e não companheiras de ninho em Acromyrmex laticeps nigrosetosus Forel, 1908 (Hymenoptera, Formicidae) $^{1}$
}

\author{
Brood recognition by workers of Acromyrmex laticeps nigrosetosus Forel, 1908 \\ (Hymenoptera, Formicidae)
}

\author{
Danival José de Souza ${ }^{2}$ Terezinha Maria Castro Della Lucia ${ }^{3}$ \\ Christine Errard ${ }^{4}$ Patrizia D'Ettorre ${ }^{5}$ Jean Luc Mercier ${ }^{6}$
}

\section{RESUMO}

Estudou-se a capacidade de discriminação de formas jovens de Acromyrmex laticeps nigrosetosus por operárias adultas da mesma subespécie. Eram oferecidas, na área de forrageamento, larvas e pupas companheiras e não companheiras de ninho, sendo quantificado o comportamento frente a essas formas jovens. Foram utilizadas colonias oriundas do município de Paraopeba, MG, Brasil, mantidas em condições de laboratório. Os resultados evidenciaram que essa subespécie não é capaz de discriminar formas jovens companheiras e não companheiras de ninho, ou seja, transportaram indiscriminadamente as formas jovens oferecidas para o interior do ninho. Também não se observou diferença significativa para o tempo de resposta de aceitação de prole companheira e não companheira de ninho.

Palavras-chave: larvas, pupas, discriminação, odor de colônia, formigas cortadeiras.

\section{ABSTRACT}

This study investigated the behavioral response (acception or rejection) of Acromyrmex laticeps nigrosetosus to their brood and to brood from different colonies of this subespecies. The four colonies used in the bioassays came from Paraopeba,
$M G$, Brazil. Workers accepted either brood from their colonies or from different colonies. There was no significant difference on the time for brood acceptance (transport to the interior of the nest) among nestmates and non-nestmates.

Key words: larvae, pupae, discrimination, odor, leaf-cutting ants.

\section{INTRODUÇÃO}

O processo típico de reconhecimento ou discriminação nos insetos sociais está baseado em pistas olfativas que constituem o odor de colônia (WILSON, 1971), comum a todos os indivíduos do ninho (BRADSHAW \& HOWSE, 1984). Nas formigas, o "odor de colônia" é usado para distinguir companheiras de ninho de outras estranhas, no reconhecimento de castas, no desencadeamento do comportamento de allogrooming (limpeza e lambeduras entre diferentes indivíduos na colônia), bem como nas secreções que estimulam a troca de alimentos (SHOREY, 1973).

A habilidade em reconhecer companheiras de ninho e distingui-las de intrusos fundamenta-se no fato de que cada colônia tem sua própria "assinatura química", uma mistura de substâncias químicas específicas e características de cada colônia

${ }^{1}$ Financiado pelo Convênio CAPES-COFECUB, N²44-1998-2000

${ }^{2}$ Biólogo, Mestrando em Entomologia , Departamento de Biologia Animal (DBA), Centro de Ciências Biológicas e da Saúde (CCB), Universidade Federal de Viçosa (UFV).

${ }^{3}$ Biólogo, Professor Titular, Doutor, DBA, CCB, UFV, 36571-000, Viçosa - MG. E-mail: tdlucia@mail.ufv.br. Autor para correspondência.

${ }^{4}$ Biólogo, Doutor, Laboratoire d'ethologie et Pharmacologie du Comportement, Université de Tours, França.

${ }^{5}$ Bioquímico, Doutor, Laboratoire d'ethologie et Pharmacologie du Comportement, Université de Tours (UT).

${ }^{6}$ Biólogo, Doutor, Laboratoire déthologie et Pharmacologie du Comportement, UT. 
(HÖLLDOBLER \& MICHENER, 1980). Acredita-se que os hidrocarbonetos cuticulares larvais são importantes informações químicas que poderiam ser utilizadas pelas operárias para distinguir larvas de outros membros da colônia (BONAVITACOUGOURDAN et al., 1993). De acordo com BOULAY et al. (2000), fatores genéticos estão envolvidos na produção de hidrocarbonetos cuticulares de cada indivíduo e há necessidade de troca destas substâncias, entre as operárias, para a manutenção do odor Gestalt. Também, feromônios nas pupas parecem estimular as operárias que cuidam da prole a ajudarem à forma jovem durante a emergência (HÖLLDOBLER, 1977).

MOREL \& VANDER MEER (1988) citam o fato de que a superfície química da forma jovem é reconhecida pelo adulto, sendo que cada estágio imaturo (ovo, larva ou pupa), produz um feromônio específico e que a atratividade das formas jovens aos adultos é devido à liberação deste feromônio.

Vários esforços vêm sendo empregados nas investigações sobre o reconhecimento de membros das colônias e nas formas desse reconhecimento em várias espécies de formigas, mas esse fenômeno ainda não foi bem elucidado, principalmente nas formigas cortadeiras. Nesse aspecto, algumas investigações foram realizadas com Atta sexdens rubropilosa por ARAÚJO et al. (1996), e com Acromyrmex subterraneus subterraneus por Viana (1996). ROBINSON \& CHERRETT (1974), trabalhando com Atta cephalotes, investigaram a atração de formas jovens às operárias, na expectativa da possibilidade do uso de feromônios de larvas e pupas como um dos componentes para aumentar a atratividade de iscas granuladas utilizadas no controle de pragas.

Acromyrmex laticeps nigrosetosus é uma subespécie de formiga cortadeira comum no território brasileiro, sendo encontrada desde a região Sul até a Norte (ANTUNES \& DELLA LUCIA, 1999). Em Minas Gerais, no município de Paraopeba, esta subespécie apresenta alta freqüência durante todo o ano e grande abundância de ninhos (ARAÚJO \& DELLA LUCIA, 1997).

Este trabalho procurou avaliar as respostas comportamentais de operárias de A. laticeps nigrosetosus a odores de larvas e pupas companheiras e não companheiras de ninho. Espera-se que os dados obtidos contribuam para maior entendimento do comportamento discriminatório nas colônias de formigas cortadeiras e sirvam de base para futuros métodos de controle de formigas-praga na agricultura, conforme, por exemplo, a utilização de feromônios de prole em iscas tóxicas granuladas.

\section{MATERIAL E MÉTODOS}

\author{
Manutenção das colônias \\ Foram utilizadas quatro colônias de $\boldsymbol{A}$.
} laticeps nigrosetosus de 2 anos de idade, coletadas no município de Paraopeba, MG, Brasil e mantidas em criação no Insetário da Universidade Federal de Viçosa, conforme descrito por DELLA LUCIA et al. (1993). Essas colônias permaneceram acondicionadas em recipientes de vidro, do tipo porta biscoito, com capacidade de $3000 \mathrm{ml}$ e $9,0 \mathrm{~cm}$ de diâmetro; as tampas eram de plástico, perfuradas e teladas, com abertura na base inferior do vidro de aproximadamente $2,0 \mathrm{~cm}$, por onde as operárias tinham acesso à área de forrageamento. Esta se constituía de uma plataforma de madeira de $0,16 \mathrm{~m} 2$, cujos pés estavam colocados numa bandeja plástica recoberta com talco nas paredes, para evitar fuga das formigas.

As colônias recebiam diariamente folhas, pétalas e flores de diferentes plantas e foram mantidas a $25-27^{\circ}$ C, U.R. acima de $75 \%$ e fotoperíodo de 12 horas.

\section{Condução dos bioensaios}

A metodologia utilizada nos bioensaios com a prole foi modificada de ARAÚJO et al. (1996). As colônias, em que eram realizados os testes, permaneciam sem receber alimentação (folhas) por um período de 24 horas, obtendo-se uma resposta mais rápida das operárias.

Foram utilizadas larvas e pupas de quatro colônias. As formas jovens eram retiradas da massa de fungo com uma pequena espátula metálica, cuidandose para não desorganizar muito a estrutura da esponja fúngica. Logo após, as formas jovens eram isoladas em placas de Petri. Todo o material utilizado para o manuseio da prole era esterilizado. Esperava-se um tempo de 15 minutos para que as operárias da colônia, de onde haviam sido retiradas as formas jovens, voltassem ao ritmo normal de forrageamento. Após este tempo, ofereceu-se uma larva da própria colônia juntamente com uma larva de outra colônia, num total de 54 repetições. Estas larvas foram oferecidas sobre lâminas de vidro, cada uma em lâmina separada, que eram marcadas na face inferior com caneta para retroprojetor, a fim de se distinguir as duas larvas. As posições das lâminas eram alteradas a cada bioensaio, evitando-se assim um condicionamento pré-imaginal das operárias. Procedimento idêntico foi feito com as pupas. No processo de discriminação contra "proles estranhas", as operárias de formigas não têm uma resposta do tipo "tudo ou nada", mas sim um comportamento preferencial pela prole de sua própria colônia 
(HÖLLDOBLER \& WILSON, 1990). As observações foram realizadas durante 20 minutos, considerando três tipos de comportamento: ninho;

1) aceitação - carregamento para o interior do

2) rejeição - carregamento para o lixo;

3) indeterminado - aglomeração em torno do material, sem carregamento. Considerou-se discriminação o ato das operárias levarem preferencialmente a prole companheira de ninho para o interior da colônia e levarem para o lixo a prole não companheira de ninho.

Para comparação dos dados, foi empregado o Teste T e do $\chi^{2}$.

\section{RESULTADOS}

\section{Discriminação de prole}

A maior parte da prole usada nos bioensaios (entre 55 e $62 \%$ ) foi aceita pela colônia receptora, independente de ser ou não companheira de ninho, pois não se verificou diferença significativa entre a aceitação de uma prole estranha e uma testemunha pelo teste do $\chi^{2}$. (Figuras 1 e 2 ).
Nos testes com larvas, o tempo médio para a colônia aceitar uma companheira de ninho (7,32 minutos) foi menor que o tempo para aceitação de uma não companheira de ninho (7,72 minutos), mas esta diferença não se mostrou significativa ( $p>0,05$, testet), logo, não houve diferença. Com pupas, verificou-se também que não houve diferença significativa $(\mathrm{p}>0.05$, teste-t) entre o tempo para aceitação de uma pupa companheira de ninho (9,32 minutos) e uma não companheira de ninho (7,78 minutos).

Vale ressaltar que, em aproximadamente $40 \%$ das vezes, o comportamento ficou indefinido, isto é, as operárias não carregaram a prole para o interior do ninho, nem as levaram para o lixo. Isso significa que tanto as operárias companheiras de ninho, quanto as não companheiras de ninho, tiveram dificuldade em discriminar ou não a sua prole.

\section{DISCUSSÃO}

Os resultados obtidos indicam que, nas condições em que o trabalho foi realizado, as operárias de A. laticeps nigrosetosus não têm a capacidade de discriminar a prole de sua própria colônia. Isso contrasta

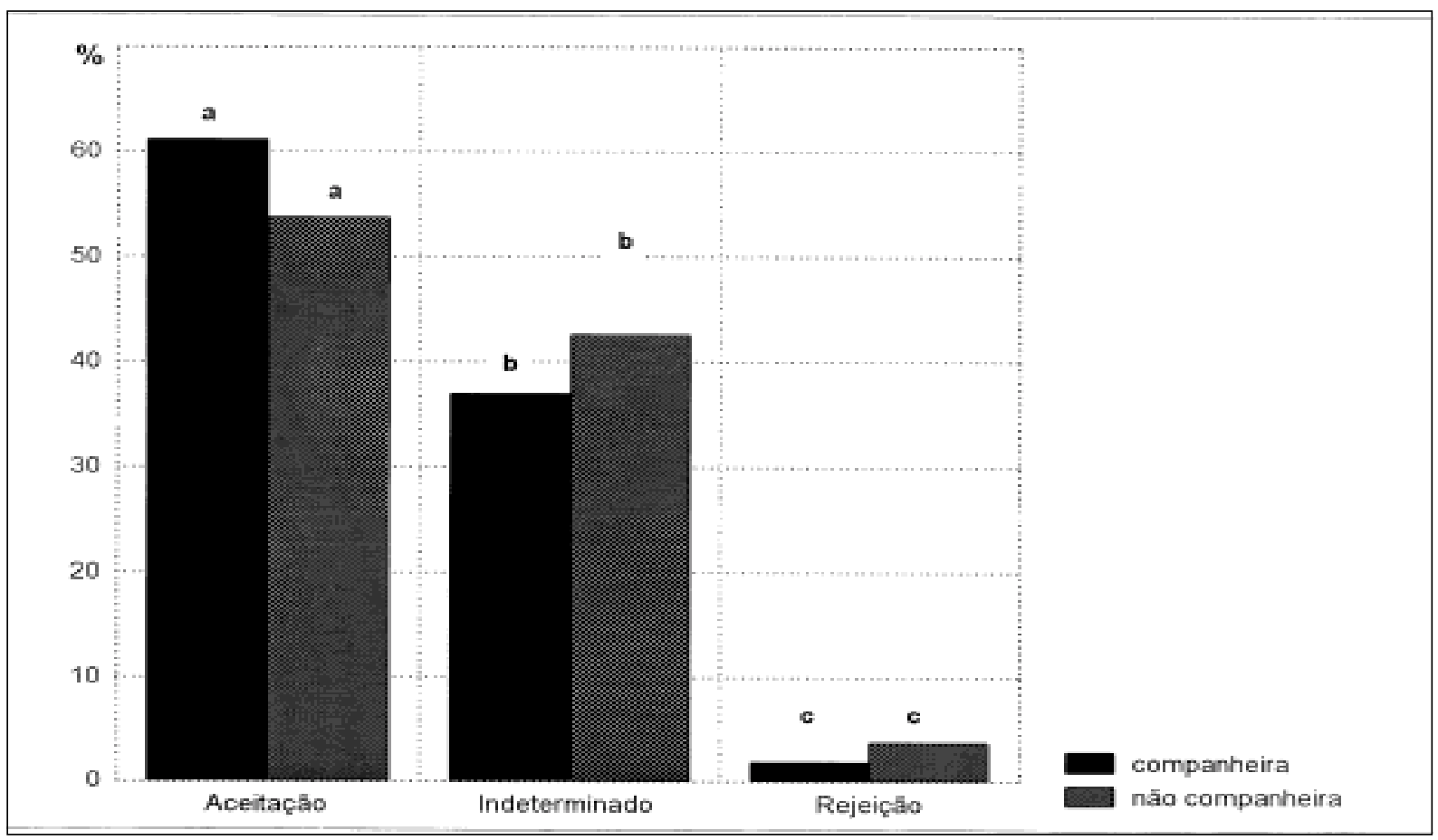

Figura 1 - Comportamento das operárias de Acromyrmex laticeps nigrosetosus frente à larvas provenientes da mesma colônia (companheiras) ou de colônias diferentes, porém da mesma espécie (não companheiras). Barras seguidas de mesma letra não diferem significativamente entre si $\left(\mathrm{p}>0.05, \chi^{2}\right)$, para cada situação. $\mathrm{n}=54$ para companheiras e $\mathrm{n}=54$ para não companheiras de ninho. 


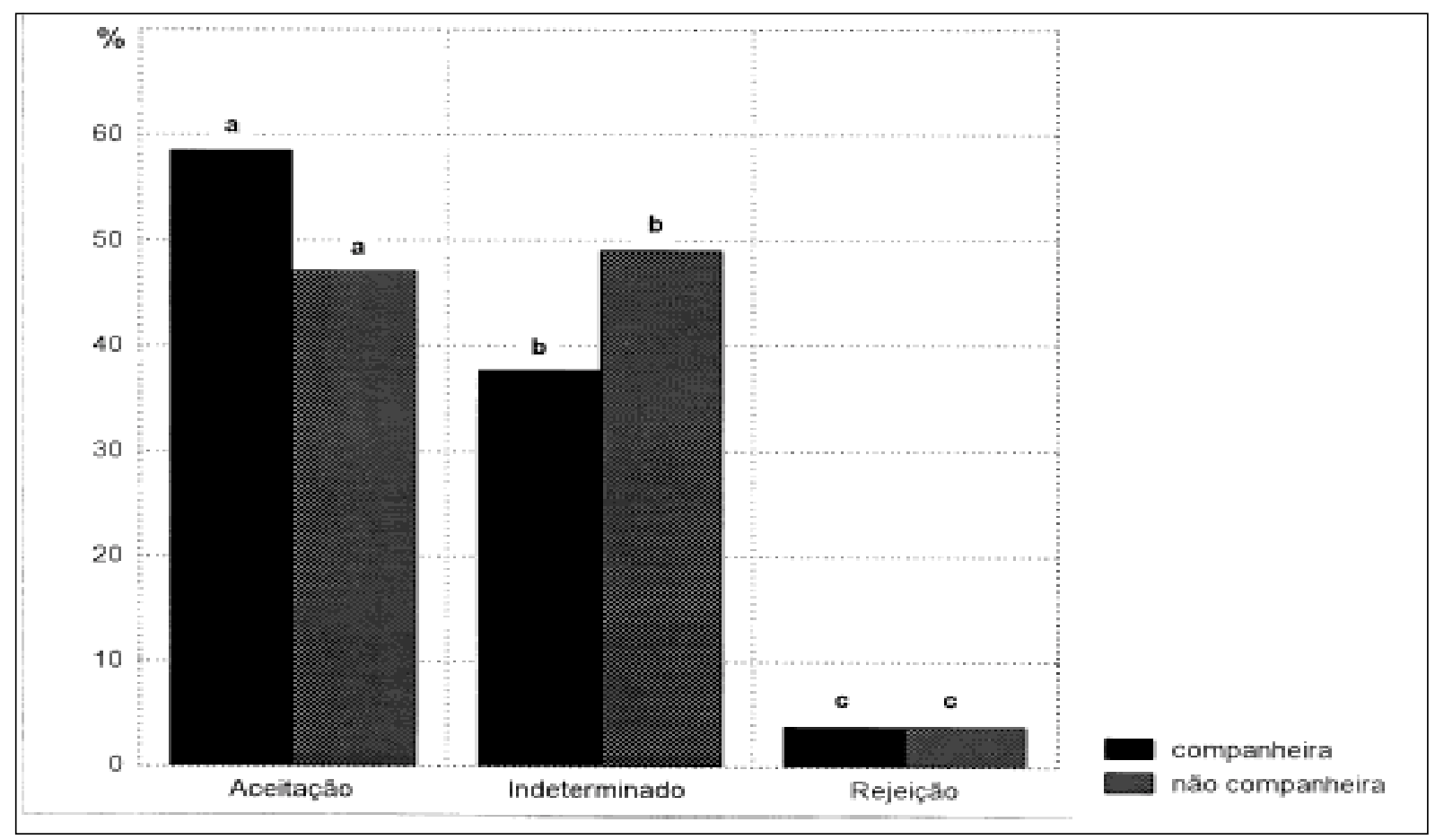

Figura 2 - Comportamento das operárias de Acromyrmex laticeps nigrosetosus frente à pupas provenientes de masma colônia (companheiras) ou de colônias diferentes, porém da mesma espécie (não companeiras). Barras seguidas de mesma letra não diferem significativamente entre si $\left(\mathrm{p}>0.05, \chi^{2}\right)$, para cada situação. $\mathrm{n}=54$ para companheiras e $\mathrm{n}=53$ para não companheiras de ninho.

com os dados obtidos no trabalho de ARAÚJO et al. (1996) com A. sexdens rubropilosa, quando os resultados dos bioensaios com larvas e pupas mostraram que as operárias foram capazes de detectar e rejeitar com a mesma eficiência a presença de formas jovens estranhas, transportando-as para o exterior (lixo), independente do tempo de isolamento.

A razão para essas diferenças está possivelmente associada ao tamanho reduzido das populações de A. laticeps nigrosetosus em contraste com as populações de $\boldsymbol{A t t a}$. É plausível assumir que a aceitação de prole de outras colônias da mesma espécie represente um artifício para aumentar a força de trabalho na colônia. Além disso, a existência de uma abertura maior dessas sociedades (no sentido de maior aceitação na sociedade) no gênero Acromyrmex (presença de múltiplas rainhas) pode estar associada a esse comportamento não discriminatório.

Um importante aspecto, pouco mencionado em discussões sobre o odor de colônia é que ele é menos distinto em espécies de formigas verdadeiramente poligínicas em relação às espécies monogínicas. Rainhas de espécies poligínicas como Formica polyctena, F. exectoides, ou Monomorium pharaonis, durante a fundação de um novo formigueiro, não são agressivas umas com as outras (HÖLLDOBLER \& MICHENER, 1980). Rainhas de colônias monogínicas, ao contrário, não toleram outras rainhas na sua vizinhança. Isto é verificado no caso de formigas cortadeiras do gênero Atta que são usualmente monogínicas. Embora as colônias de $A$. laticeps nigrosetosus coletadas até então e utilizadas nos testes, possuíssem apenas uma rainha, em ninhos de Acromyrmex subterraneus subterraneus e Acromyrmex subterraneus molestans (DELLA LUCIA \& VILELA, 1986) e Acromyrmex subterraneus brunneus (DELABIE, 1989) foram encontradas múltiplas rainhas.

Ressaltadas estas diferenças entre os gêneros Atta e Acromyrmex pode-se especular sobre a ausência da capacidade de discriminação verificada em A. laticeps nigrosetosus. A poliginia é um traço marcante do gênero Acromyrmex, sendo que colônias poligínicas tendem a apresentar menor grau de agressividade e maior tolerância a ninhos vizinhos (HÖLLDOBLER \& MICHENER, 1980).

A questão levantada se existe ou não um feromônio presente nas formas jovens de formigas foi 
amplamente discutida por MOREL \& VANDER MEER (1988). Segundo estes autores, vários problemas surgem na tentativa de demonstrar conclusivamente que a prole produz feromônios. Os mais importantes são a espécie-especificidade de um feromônio, a utilização de bioensaios adequados, a existência de controles seguros, que incluem alimento e controle de reconhecimento de companheiras de ninho, a quantidade de material usado para obter uma resposta positiva e as técnicas químicas empregadas (MOREL \& VANDER MEER, 1988).

As colônias de A. laticeps nigrosetosus utilizadas neste trabalho são oriundas da mesma região, sendo coletadas próximas uma das outras. As condições em que foram criadas em laboratório foram bastante homogêneas. Considerando-se o modelo de odor de colônia Gestalt (BOULAY et al., 2000), pode-se aventar a hipótese de que as semelhantes condições a que estas colônias permanecem submetidas em laboratório tracem um perfil de hidrocarbonetos cuticulares muito semelhante entre as diferentes colônias, o que explicaria a ausência de discriminação verificada neste experimento.

\section{CONCLUSÃO}

Operárias de Acromyrmex laticeps nigrosetosus não discriminam larvas e pupas de colônias diferentes da sua própria.

\section{AGRADECIMENTOS}

Os autores agradecem ao Doutorando em Entomologia, Márcio da Silva Araújo; à professora Dra. do Departamento de Biologia Geral da UFV, Mara Garcia Tavares; à Mestranda em Entomologia, Ethel Fernandes Petternelli, pelas valiosas sugestões no manuscrito. Agradecem, ainda, ao Sr. Manoel Ferreira, pela colaboração na execução dos trabalhos.

\section{REFERÊNCIAS BIBLIOGRÁFICAS}

ANTUNES, E. C.; DELLA LUCIA, T. M. C. Consumo foliar em Eucalytus urophylla por Acromyrmex laticeps nigrosetosus FOREL (Hymenoptera, Formicidae). Ciência e Agrotecnologia, v.23, p.298-211, 1999.

ARAÚJO, M. S. et al. Discriminação da prole por operárias companheiras e não companheiras de ninho em Atta sexdens rubropilosa Forel, 1908 (Hymenoptera, Formicidae). Revista Brasileira de Entomologia, v.40, n.1, p.101-104, 1996.

ARAÚJO, M. S.; DELLA LUCIA, T. M. C. Caracterização de ninhos de Acromyrmex laticeps nigrosetosus Forel, em povoamento de eucalipto em Paraopeba, MG. Anais da Sociedade Entomológica do Brasil, v.26, n.1, p.205-207, 1997.

BOULAY, R. et al. Camponotus fellah colony integration: worker individuality necessitates frequent hydrocarbon exchanges. Animal Behavior, v.59, p.1127-1133, 2000.

BONAVITA-COUGOURDAN, A.; CLEMENT, J. L.; LANGE, C. Functional subcaste discrimination (foragers and brood-tenders) in the ant Camponotus vagus Scop: polymorphism of cuticular hydrocarbon patterns. Journal of Chemical Ecology, v.9, n.7, p.1461-1477, 1993.

BRADSHAW, J. W. S.; HOWSE, P. E. Sociochemicals of ants. In: BELL, W. J.; CARDÉ, R. T. Chemical ecology of insect. London : Chapman and Hall, 1984. p.429-473.

DELABIE, J. H. C. Observações sobre a ocorrência de poliginia em colônias de Acromyrmex subterraneus brunneus Forel, 1893 (Formicidae, Myrmcinae, Attini) em cacauais. Anais da Sociedade Entomológica do Brasil, v.18, n.1, p.193-197, 1989.

DELLA LUCIA, T. M. C. et al. Criação de formigas cortadeiras em laboratório. In: DELLA LUCIA, T. M. C. As formigas cortadeiras. Viçosa : Folha de Viçosa, 1993. p.1-3.

DELLA LUCIA, T. M. C.; VILELA, E. F. Ocorrência de poliginia em Acromyrmex subterraneus subterraneus Forel, 1893 (Hymenoptera: Formicidae). Anais da Sociedade Entomológica do Brasil, v.15, n.2, p.379-380, 1986.

HÖLLDOBLER, B. Comunication in social hymenoptera. In: SEBEOK, T. A. How animals communicate. Bloomington: Indiana University, 1977. p.418-471.

HÖLLDOBLER, B.; MICHENER, C. D. Mechanisms of identification and discrimination in social hymenoptera. In: MARKL, H. Evolution of social behavior: hypotheses and empirical tests. Weinheim : Chemie Gmbh, 1980. p.35-38.

HÖLLDOBLER, B.; WILSON, E.O. The ants. Cambridge : The Belknap, 1990. 732p.

MOREL, L.; VANDER MEER, R. K. Do ant brood pheromones exist? Annais of Entomological Societ American, v.81, n.5, p.705$710,1988$.

ROBINSON, S. W.; CHERRETT, J. M. Laboratory investigations to evaluate the possible use of pheromones of the leaf-cutting ant Atta cephalotes (L.) (Formicidae: Attini) as a component in na attractive bait. Bulletin of Entomological Research, v.63, n.3, p.519-529, 1974.

SHOREY, H. H. Behavioral responses to insect pheromones. Annais Review of Entomology, v.18, p.349-380, 1973.

VIANA. A. M. M. La reconnaissance coloniale du couvain et du champignon chez la fourmi champignonniste Acromyrmex subterraneus subterraneus. 1996. 136f. Tese (Doutorado em Biologia dos Organismos) - Curso de Pós-graduação em Biologia dos Organismos, Université Paris XIII.

WILSON, E. O. The insect societies. Cambridge : Harvard University, 1971. 